\title{
Correction to: Direct oral anticoagulants in patients with severe inherited thrombophilia: a single-center cohort study
}

\author{
Joanna Zuk ${ }^{1,2} \cdot$ Elzbieta Papuga-Szela $^{1} \cdot$ Lech Zareba $^{3} \cdot$ Anetta Undas $^{1,4}$ (D) \\ Published online: 5 January 2021 \\ C Japanese Society of Hematology 2021
}

\section{Correction to: International Journal of Hematology https://doi.org/10.1007/s12185-020-03012-7}

In the original publication of the article, the following errors were corrected.

In the abstract, in line 5, there is one space missing, instead of "apixabanin 14 (25\%)", it should read as "apixaban in $14(25 \%)$ ".

In the Laboratory Investigations section, the reference range for total PS antigen was incorrectly published (5-140\%). The correct reference range for total PS antigen is: $75-140 \%$.

In the Follow-up section of Results, there was an error in the description of the third patient with VTE recurrence. The patient should be presented as follows: The third patient, a 56-year-old woman with PS deficiency, FVL mutation and coexisting comorbidities, was hospitalized due to pneumonia while on rivaroxaban for 9 months (...) She was treated with enoxaparin for 4 weeks and then wanted to return to the treatment with rivaroxaban. During 10 months' followup, no recurrent VTE was observed, but she reported slight hematuria and easy bruising. Treatment with rivaroxaban was continued.
In the description of last case, there was an error in the mutation type, instead of heterozygous FVL, it was heterozygous prothrombin 20210A mutation.

In the Table 2, in the description of the third patient, in the column "thrombophilia", the mutation should read as "FVLGA" (instead of FII20210A), in the column "current DOAC" rivaroxaban should appear instead of "apixaban", as well as, in the column "event time", instead of 6 , there should appear 9 months.

In the Table 3, in the description of the sixth patient, in the column "thrombophilia", the mutation should read as "FVLGA" (instead of FII20210A) and in the column "current DOAC" rivaroxaban should appear instead of "apixaban.

These corrections now appear in the article online.

Publisher's Note Springer Nature remains neutral with regard to jurisdictional claims in published maps and institutional affiliations.

The original article can be found online at https://doi.org/10.1007/ s12185-020-03012-7.

Anetta Undas

mmundas@cyf-kr.edu.pl

1 Institute of Cardiology, John Paul II Hospital, Jagiellonian University Medical College, Krakow, Poland

2 Department of Internal Medicine, Jagiellonian University Medical College, Krakow, Poland

3 Faculty of Mathematics and Natural Sciences, University of Rzeszow, Rzeszow, Poland

4 Institute of Cardiology, Jagiellonian University Medical College, Krakow, Poland 

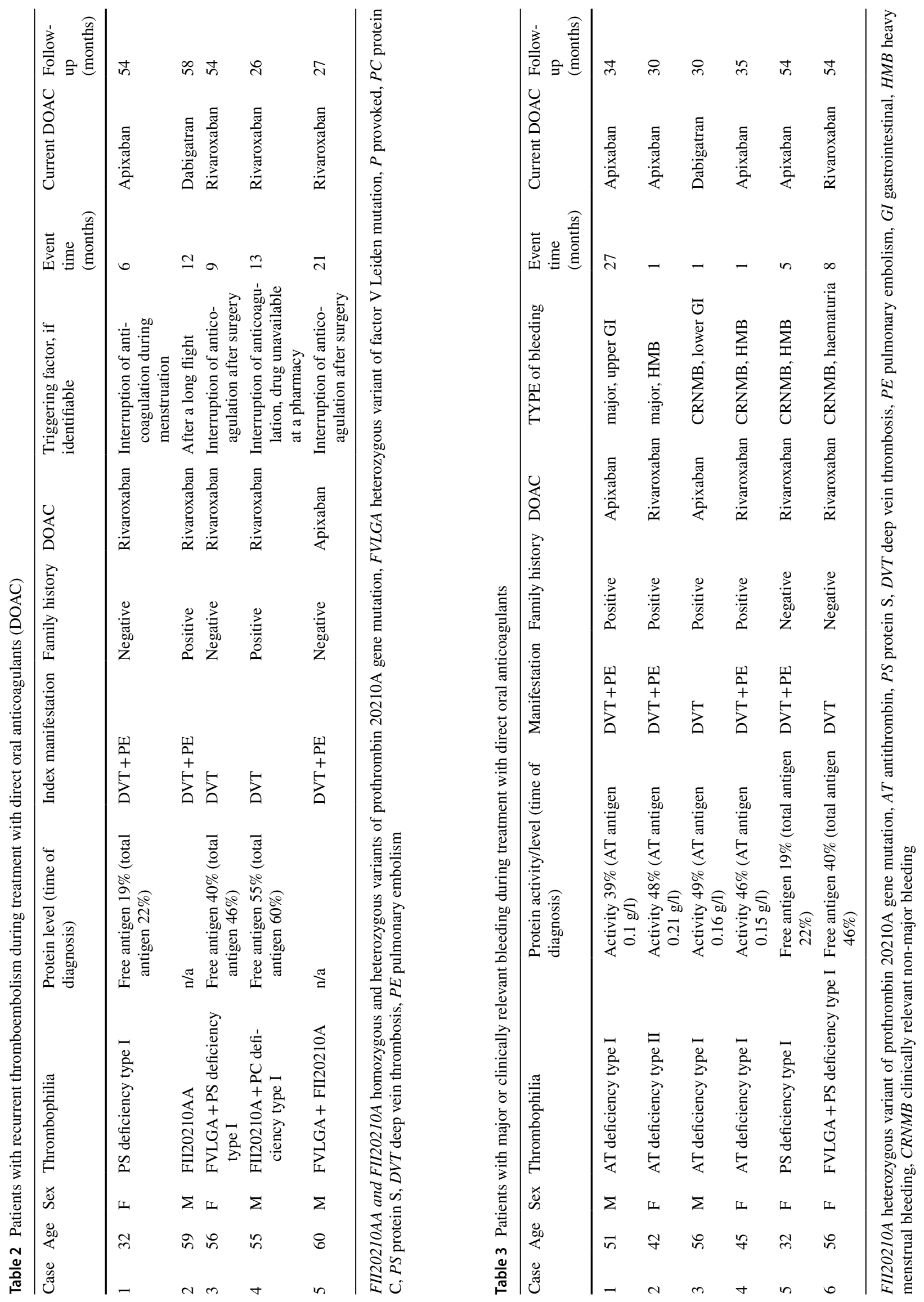